\title{
Mapping the multicausality of Alzheimer's disease through group model building
}

\author{
Jeroen F. Uleman (D) - René J. F. Melis • Rick Quax • Eddy A. van der Zee • \\ Dick Thijssen • Martin Dresler • Ondine van de Rest • Isabelle F. van der Velpen • \\ Hieab H. H. Adams • Ben Schmand • Inge M. C. M. de Kok • Jeroen de Bresser • \\ Edo Richard • Marcel Verbeek • Alfons G. Hoekstra • Etiënne A. J. A. Rouwette • \\ Marcel G. M. Olde Rikkert
}

Received: 28 April 2020 / Accepted: 6 July 2020 / Published online: 11 August 2020

(C) The Author(s) 2020

\begin{abstract}
Alzheimer's disease (AD) is a complex, multicausal disorder involving several spatiotemporal scales and scientific domains. While many studies focus on specific parts of this system, the complexity
\end{abstract}

Electronic supplementary material The online version of this article (https://doi.org/10.1007/s11357-020-00228-7) contains supplementary material, which is available to authorized users.

J. F. Uleman $(\bowtie) \cdot$ M. G. M. Olde Rikkert

Department of Geriatric Medicine, Radboudumc

Alzheimer Center, Donders Institute for Brain,

Cognition and Behaviour, Radboud University

Medical Center, Reinier Postlaan 4,

6525GC, Nijmegen, The Netherlands

e-mail: jeroen.uleman@radboudumc.nl

J. F. Uleman · R. J. F. Melis · A. G. Hoekstra

Institute for Advanced Study, Amsterdam, The Netherlands

R. Quax · A. G. Hoekstra

Computational Science Lab, Faculty of Science, University

of Amsterdam, Amsterdam, The Netherlands

E. A. J. A. Rouwette

Institute for Management Research, Radboud University, Nijmegen, The Netherlands

\section{R. J. F. Melis}

Department of Geriatric Medicine, Radboud Institute for Health Sciences, Radboud University Medical Center, Nijmegen, The Netherlands

E. A. van der Zee

Molecular Neurobiology, Groningen Institute for Evolutionary Life Sciences (GELIFES), University of Groningen, Groningen, The Netherlands of $\mathrm{AD}$ is rarely studied as a whole. In this work, we apply systems thinking to map out known causal mechanisms and risk factors ranging from intracellular to psychosocial scales in sporadic AD. We report on

\section{Thijssen}

Department of Physiology, Radboud Institute for Health Sciences, Radboud University Medical Center, Nijmegen, The Netherlands

D. Thijssen

Liverpool John Moores University, Liverpool,

United Kingdom

M. Dresler

Donders Institute for Brain, Cognition and Behaviour, Radboud University Medical Center, Nijmegen,

The Netherlands

O. van de Rest

Division of Human Nutrition and Health, Wageningen University, Research, Wageningen, The Netherlands

I. F. van der Velpen Department of Epidemiology, Department of Radiology and Nuclear Medicine, Erasmus MC, University Medical Center Rotterdam, Rotterdam, The Netherlands

B. Schmand

Department of Psychology, University of Amsterdam, Amsterdam, The Netherlands

I. M. C. M. de Kok

Department of Public Health, Erasmus MC, University Medical Center Rotterdam, Rotterdam, The Netherlands 
the first systemic causal loop diagram (CLD) for AD, which is the result of an interdisciplinary group model building (GMB) process. The GMB was based on the input of experts from multiple domains and all proposed mechanisms were supported by scientific literature. The CLD elucidates interaction and feedback mechanisms that contribute to cognitive decline from midlife onward as described by the experts. As an immediate outcome, we observed several non-trivial reinforcing feedback loops involving factors at multiple spatial scales, which are rarely considered within the same theoretical framework. We also observed high centrality for modifiable risk factors such as social relationships and physical activity, which suggests they may be promising leverage points for interventions. This illustrates how a CLD from an interdisciplinary GMB process may lead to novel insights into complex disorders. Furthermore, the CLD is the first step in the development of a computational model for simulating the effects of risk factors on $\mathrm{AD}$.

Keywords Systems thinking - Alzheimer's disease · Causal loop diagram · Multicausal · Complexity · Group model building $\cdot$ Centrality

\section{Introduction}

Alzheimer's disease is multicausal

Alzheimer's disease (AD) is a complex disorder with a multicausal etiology that remains difficult to elucidate

\author{
J. de Bresser \\ Department of Radiology, Leiden University Medical Center, \\ Leiden, The Netherlands \\ E. Richard \\ Department of Neurology, Donders Institute for Brain, \\ Cognition and Behaviour, Radboud University Medical \\ Centre, Nijmegen, The Netherlands \\ M. Verbeek \\ Departments of Neurology and Laboratory Medicine, \\ Donders Institute for Brain, Cognition and Behaviour, Radboud \\ University Medical Centre, Nijmegen, The Netherlands \\ H. H. H. Adams \\ Department of Clinical Genetics, Erasmus MC, University \\ Medical Center Rotterdam, Rotterdam, The Netherlands \\ H. H. H. Adams \\ Department of Radiology and Nuclear Medicine, Erasmus MC, \\ University Medical Center Rotterdam, Rotterdam, \\ The Netherlands
}

despite the many and diverse research efforts, which focus on various causal mechanisms. For instance, the most prominent causal hypothesis concerns the aggregation of amyloid-beta protein in the brain, leading to the formation of senile plaques and neuronal dysfunction (Hardy and Selkoe 2002; Karran et al. 2011). However, the relationship between this amyloid cascade and the onset and progression of $\mathrm{AD}$ lacks specificity (Mortimer 2012) and weakens with increasing age and frailty (Savva GM et al. 2009; Wallace et al. 2019). Furthermore, clinical trials based on this hypothesis have not yet yielded effective diseasemodifying treatments (Cummings et al. 2019; Karran et al. 2011). One may thus tentatively conclude that while the amyloid cascade appears to play a role in $\mathrm{AD}$, it is far from the complete story (Pimplikar 2009).

Indeed, in recent years, researchers argue that unraveling this complexity at the systemic level may be crucial for the development of efficacious treatments (Pomorska and Ockene 2017; Tang et al. 2019; Rollo et al. 2016). This is supported by many additional mechanisms that are probably involved in the etiology of $\mathrm{AD}$, such as glucose metabolism and oxidative stress (Butterfield and Halliwell 2019; Nunomura et al. 2006), vascular dysfunction (Sweeney et al. 2019), and inflammation (Newcombe et al. 2018; Heneka et al. 2015). This multicausality is increasingly recognized (Fotuhi et al. 2009; Sweeney et al. 2019), but a comprehensive understanding of the interactions between these causes is lacking. Such an overarching understanding of $\mathrm{AD}$ requires intense interdisciplinary collaboration between various traditional scientific disciplines that now largely focus on a single scale or mechanism (Kuljis 2009; van Dijk et al. 2015), supported by methodologies that are largely novel in this field, originating from complexity science and the study of complex adaptive systems (Braithwaite et al. 2018).

Applying systems thinking to Alzheimer's disease

Systems thinking offers a methodology to realize such a holistic, interdisciplinary approach. In this way, it helps to understand the behavior of complex adaptive systems such as organs and organisms, and may thus also be useful in disentangling the interaction and feedback mechanisms that lead to cognitive decline. It offers validated methods for identifying causal mechanisms and the interactions between them. 
Unsurprisingly, systems thinking is being increasingly recognized and utilized in biomedical and neuroscience literature (Wittenborn et al. 2016; Kenzie et al. 2018; Vandenbroeck et al. 2007).

An important concept in systems thinking is the causal loop diagram (CLD), which is a conceptual model of relevant mechanisms and their interactions. In this paper, we report on the first systemic CLD for $\mathrm{AD}$, which was realized using group model building (GMB). The CLD comprises the combined conceptual model of an extensive interdisciplinary group of $\mathrm{AD}$ researchers and computational modelers, checked against the scientific literature. We do not present the CLD as a complete representation of reality but rather as a summary of knowledge, agreed upon by this specific group of experts, which helps elucidate central processes involved in the onset and progression of $\mathrm{AD}$.

\section{Methods}

Causal loop diagram

CLDs visualize the known or assumed causal structure of a system and consist of variables and connections drawn as arrows between the variables. These connections have specific directions, in line with the underlying causal relationships, and typically have positive or negative polarity (Bala et al. 2017). A positive connection (+) implies that when the causal variable changes in one direction, the variable it connects to changes in the same direction; a negative connection (-) implies that when the causal variable changes in one direction, the variable it connects to changes in the opposite direction. A direct connection between two variables implies an effect that does not go via any of the other variables in the CLD.

As the name suggests, an important feature of CLDs is the presence of feedback loops. Feedback mechanisms are important drivers of the nonlinear behavior of dynamical systems (Forrester 2009; Littlejohns et al. 2018) and can reinforce or balance the impact of stimuli. Reinforcing loops have a selfstrengthening effect and can push the system out of balance, whereas balancing loops have a self-limiting effect and promote equilibrium restoration and thus support homeostasis. Identifying reinforcing loops may be particularly important for understanding the amplification of inter-individual differences in risk profiles, which can result in substantial heterogeneity (Sterman 2000) in terms of onset and progression of AD.

Feedback loops with only two variables are "direct," while loops consisting of more than two variables are defined as "indirect." When the variables in a loop all occur in the same characteristic spatial scale (e.g., cellular), the loop is referred to as "within-scale." When the variables in the loop occur in different spatial scales, the loop is referred to as "cross-scale." Identifying cross-scale loops is of particular interest because they can be easily overlooked when studying the system exclusively from one spatial scale or scientific domain. From a systems perspective, all known and hypothesized causal relationships between variables should be taken into account, even those that are separated by spatial or temporal scales (Forrester 1971).

Group model building

Group model building (GMB) is a participatory method for involving experts in developing conceptual or computational models (Vennix 1999; Andersen et al. 2007; Hovmand et al. 2012). In GMB, the mental models and assumptions of experts are elicited and captured in a shared model, which is the result of consensus in the group (Bérard 2010; Vennix 1996). The resulting model is a summary of explicit, tested, and integrated knowledge of the group.

We organized two GMB sessions with two weeks in between, specifically aimed at developing a CLD. We aimed to include a sufficiently large variety of expertise while ensuring some overlap. To this end, we formulated an expertise table (see supplementary materials). The group consisted of thirteen domain experts from a wide scope of $\mathrm{AD}$ research and two experts in complexity research and computational modeling. The expressed purpose of our GMB sessions was to structurally explain the difference between cognitive decline trajectories in sporadic AD compared with normal aging, starting from midlife. We defined AD using clinical criteria (McKhann et al. 2011) and focused on sporadic $\mathrm{AD}$, meaning that we did not take into account the genes associated with familial AD: PSEN1, PSEN2, and APP (Dorszewska et al. 2016).

During the GMB sessions, a facilitator (EAJAR) guided the group discussions and a computational modeler (JFU) sketched resulting versions of the CLD 
on a screen using Vensim PLE (Version 7.3.5) (Vensim 2019). We alternated between phases of divergence and convergence. During divergence, the nominal group technique (Gustafson et al. 1986) was applied, during which the experts individually wrote down variables to add to the CLD. These variables were then collected and displayed on the screen for all participants to see. During convergence, debate was encouraged between experts to reach a consensus about the addition or deletion of certain variables or connections. Any proposed additions were discussed in the group and only added to the CLD when objections were resolved and a consensus was reached. During the second session, three subgroups were formed based on the main research topics of the experts (see the "Results" section). The subgroups were tasked with checking the parts of the model most closely connected to their area of expertise. The changes made by these subgroups were presented and discussed with the rest of the group.

After each GMB session, a summary report containing all results, considerations, and discussion points was sent to the participating experts. The experts were then asked to provide feedback on the reports of both sessions. After the final session, the experts provided scientific evidence in the form of literature references for each of the connections in the CLD together with the modeling team. This resulted in several additional changes to the CLD, which were communicated back to and accepted by the group.

\section{Network analysis}

The causal connections in the resulting CLD can be interpreted to form a network structure. For network processes, a typical initial analysis is to identify structurally important, or "central", variables. Such an analysis can also help pinpoint central drivers of disease in CLDs (McGlashan et al. 2016). To this end, we calculated the betweenness centrality (BC) and closeness centrality $(\mathrm{CC})$ for each of the variables in the CLD.

In a CLD, shortest paths exist between each pair of variables, which correspond to sequences of connections that contain the least number of mediating variables in the causal pathway. If mediating variables are seen as possible points of interfering with, or adding noise to, the causal pathway then it follows that shortest paths tend to be the strongest causal pathways.
As such, measures that are defined in terms of shortest paths, such as BC and CC (Brandes et al. 2016), could be informative of the importance of factors in a causal way. In particular, variables with high BC lie on many of the shortest paths between other variables, making them potentially important connectors (Ahmed 2017). That is, mediating multiple different causal pathways simultaneously. These variables could be succinct points for diagnosing aberrant system dynamics and also serve as potential targets for interventions. Complementarily, variables with high closeness have shortest paths to many other variables in the CLD with a short distance, i.e., few mediating variables, rendering them potentially efficient spreaders of information (Ahmed 2017). These variables could thus also be good starting points for interventions.

The normalized BC of variable $v$ is calculated using Eq. 1, where $N$ is the number of variables in the CLD, $\sigma(s, t)$ is the number of shortest paths between variables $s$ and $t$, and $\sigma(s, t \mid v)$ is the number of those shortest paths that pass through variable $v$.

$\mathrm{BC}_{v}=\frac{1}{(N-1)(N-2)} \sum_{s, t} \frac{\sigma(s, t \mid v)}{\sigma(s, t)}$

The CC of variable $v$ is calculated using Eq. 2, where $n-1$ is the number of reachable variables and $d(v, u)$ is the distance of the shortest path from variable $v$ to variable $u$.

$\mathrm{CC}_{v}=(n-1) \frac{1}{\sum_{u=1}^{n-1} d(v, u)}$

In order to test the robustness of these measures to possible errors in the structure of the CLD, we created 1000 alternative CLDs, each with 5 mutations in them compared with the original. These mutations were equiprobable and consisted of either a random rewiring (e.g., some connection A->B is replaced by connection $\mathrm{A}->\mathrm{C}$ ) or the random addition or deletion of a connection. Centrality measures were calculated for each of these alternative CLDs and the interquartile ranges of the resulting distributions were used to construct error bars.

Another way of assessing the behavior of the system represented by a CLD is to study its feedback loops, which can result in nonlinear influences of the variables (Forrester 2009). Important within- and cross-scale feedback loops are therefore visualized in the CLD and the potential relevance of several examples to $\mathrm{AD}$ will be discussed. 


\section{Results}

The CLD shown in Fig. 1 is a graphical depiction of the combined multidisciplinary knowledge of fifteen experts. These experts proposed, discussed, and agreed on 38 variables and 150 connections between them. The variables in the CLD were divided into three categories: brain health, physical health, and psychosocial health. These categories correspond to the subgroups in which the experts were divided and can be found in the supplementary material, together with definitions of each of the variables as well as supporting evidence for the connections. An interactive visualization of the CLD was created in Kumu (2019) which can be found https://cldforad.kumu.io/mapping-the-complexmulticausality-in-alzheimers-disease?token $=16 \mathrm{CvrnW}$ TeDcLkHR1.

\section{Variable centrality}

The centrality measures for each of the variables in the CLD are given in Fig. 2. Spearman's rankcorrelation coefficient between the two measures (BC and $\mathrm{CC}$ ) is $\rho=0.50$. Neuronal dysfunction has a very high $\mathrm{BC}$ and is clearly an important variable in the
CLD, connecting many pathophysiological variables to structural brain damage, daily and cognitive functioning. The central position of neuronal dysfunction (and neuronal loss, which is the ultimate state of neuronal dysfunction) in the CLD may indicate its role as a final common pathway that is implicated in $\mathrm{AD}$ : at the level of the brain most mechanisms cause cognitive decline via neuronal dysfunction. Furthermore, at the interface of the circulation and brain, cerebral endothelial dysfunction appears to have an important role connecting risk factors such as physical inactivity, obesity, dyslipidemia, diabetes, and hypertension to brain pathology. That being said, the experts also agreed on paths to neuronal dysfunction that do not go via cerebral endothelial dysfunction, such as effects of smoking and alcohol use via DNA methylation (Corley et al. 2019; Xu et al. 2019). Lifestyle factors like social relationships, physical activity, healthy dietary patterns, sleep quality, and depressive symptoms have high $\mathrm{BC}$ as well as $\mathrm{CC}$, indicating that they may also be important drivers of vascular and neuronal dysfunction. Furthermore, sleep deprivation and depressive symptoms can exert direct effects that result in cognitive impairment (Alhola and Polo-Kantola 2007; Rock et al. 2014).

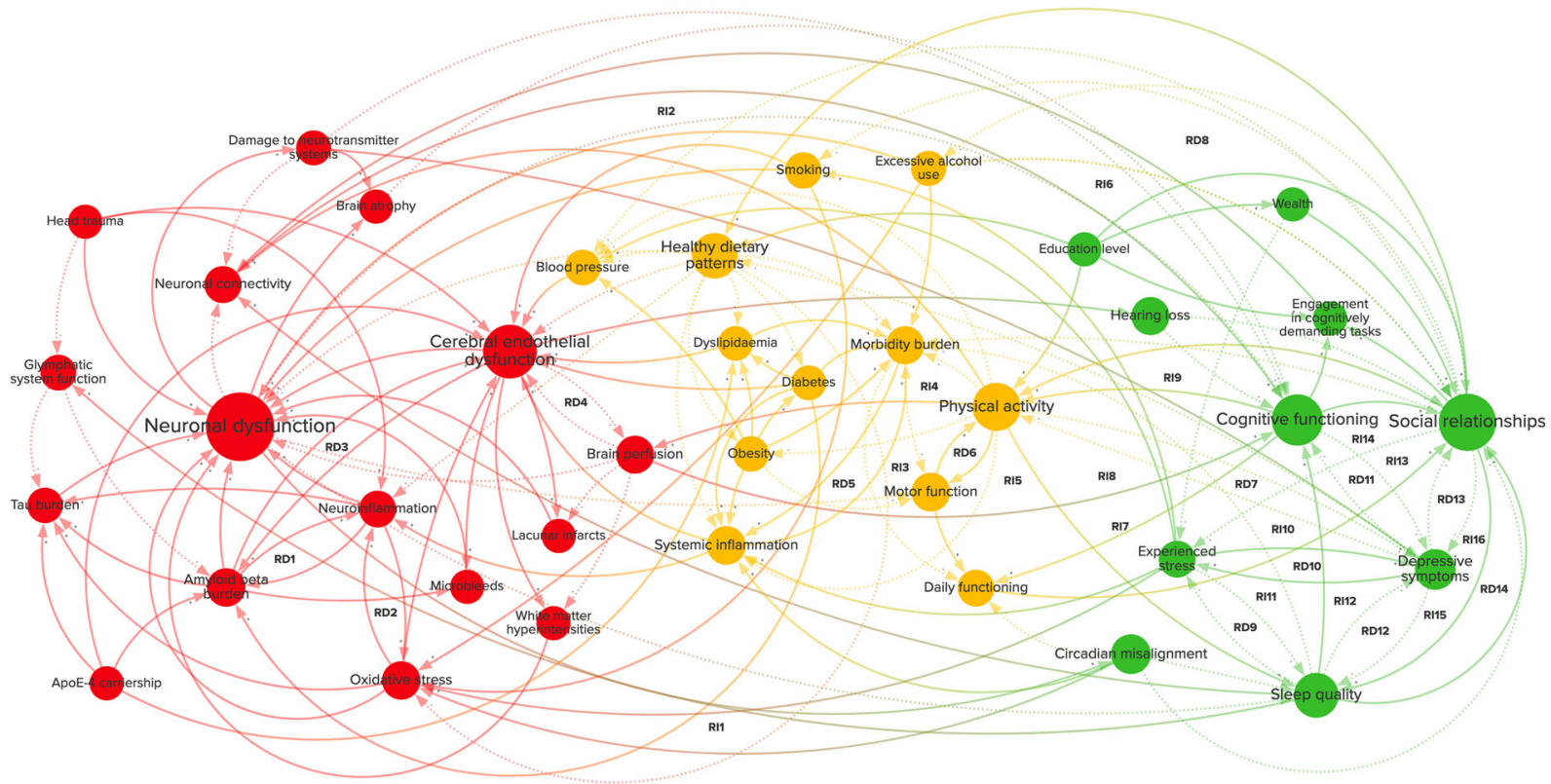

Fig. 1 Causal loop diagram of sporadic Alzheimer's disease. The diagram contains related variables and causal connections between them. The diagram is divided into variables related to brain health (red), physical health (yellow), and psychosocial health (green). A positive connection $(+$, solid line) represents an effect in the same direction, whereas a negative connection (, dotted line) represents an effect in the opposite direction. The size of the variables is scaled by their betweenness centrality. RD1-14 and RI1-16 represent direct and indirect reinforcing feedback loops 


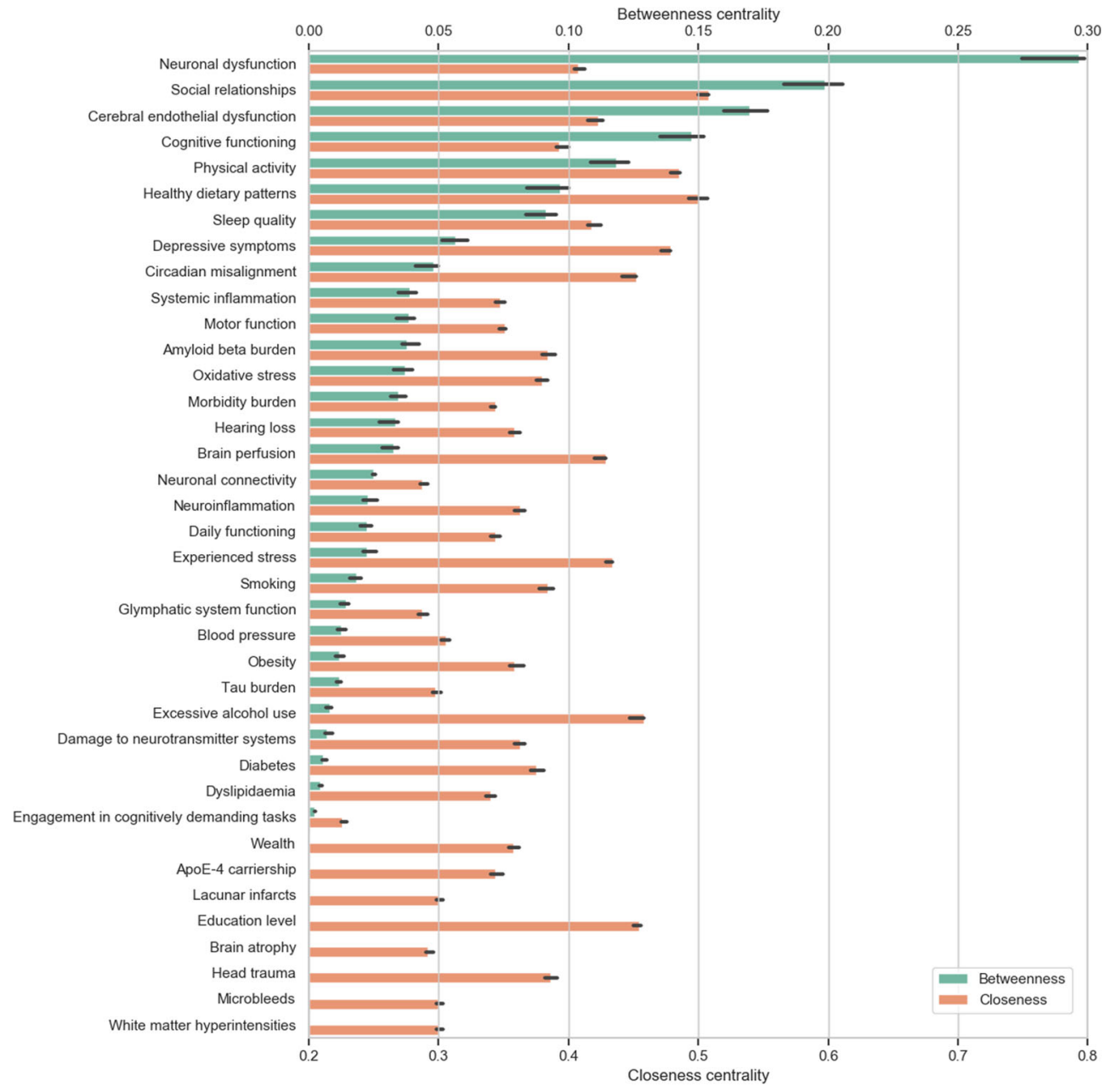

Fig. 2 The betweenness and closeness centralities of the variables in the causal loop diagram (Fig. 1). The error bars represent the interquartile range of 1000 mutated diagrams with each 5 random rewirings, additions of deletions

\section{Exogenous variables}

Education level, head trauma, and apoE-4 carriership are exogenous to the CLD and thus have a $\mathrm{BC}$ of 0 . The CC of these variables, however, is relatively high, especially of education level, suggesting that it is an important contributor to cognitive decline (Wilson et al. 2009). Exogenous variables influence the system without being influenced by it. They can be seen as part of the context of an individual and their history. They can also be seen as entry points into the system. ApoE-4 carriership might, for example, play a role in dyslipidemia (Marais 2019) and could thereby contribute to $\mathrm{AD}$ pathogenesis.

Within and cross-scale feedback loops

Several feedback loops have been identified in the CLD and are annotated in Fig. 1. An overview of the feedback loops is also given in Table 1. All the direct reinforcing loops have been denoted as RD1RD14. Similarly, indirect reinforcing loops, limited to 
three variables, have been denoted as RI1-RI6. None of these loops are balancing, suggesting that balancing loops may be of less importance than reinforcing loops for understanding cognitive decline trajectories in sporadic AD.

Within-scale loops can significantly impact local pathological processes and may fall within the scope of one scientific domain. For example, at the level of the brain, loop RD1 may aggravate neuronal dysfunction as aggregates of amyloid-beta protein may activate microglia, leading to increased release of TNF- $\alpha$, which can inhibit the phagocytosis of amyloid-beta (Tejera and Heneka 2016).

An example of a cross-scale loop is RI1. Oxidative stress can lead to an impairment of glucose metabolism and thereby to neuronal dysfunction

Table 1 Feedback loops in the causal loop diagram (Fig. 1)

\begin{tabular}{|c|c|c|c|}
\hline Loop & 1st variable & 2nd variable & 3rd variable \\
\hline RD1 & Amyloid beta burden & Neuroinflammation & - \\
\hline RD2 & Oxidative stress & Neuroinflammation & - \\
\hline RD3 & Amyloid beta burden & Cerebral endothelial dysfunction & - \\
\hline RD4 & Brain perfusion & Cerebral endothelial dysfunction & - \\
\hline RD5 & Systemic inflammation & Morbidity burden & - \\
\hline RD6 & Motor function & Physical activity & - \\
\hline RD7 & Physical activity & Depressive symptoms & - \\
\hline RD8 & Excessive alcohol use & Social relationships & - \\
\hline RD9 & Sleep quality & Experienced stress & - \\
\hline RD10 & Depressive symptoms & Experienced stress & - \\
\hline RD11 & Depressive symptoms & Cognitive functioning & - \\
\hline RD12 & Depressive symptoms & Sleep quality & - \\
\hline RD13 & Depressive symptoms & Social relationships & - \\
\hline RD14 & Sleep quality & Social relationships & - \\
\hline RI1 & Oxidative stress & Neuronal dysfunction & Circadian misalignment \\
\hline RI2 & Physical activity & Neuronal connectivity & Cognitive functioning \\
\hline RI3 & Obesity & Motor function & Physical activity \\
\hline RI4 & Morbidity burden & Motor function & Physical activity \\
\hline RI5 & Brain perfusion & Physical activity & Cognitive functioning \\
\hline RI6 & Neuronal connectivity & Cognitive functioning & Engagement in cognitively demanding tasks \\
\hline RI7 & Morbidity burden & Daily functioning & Social relationships \\
\hline RI8 & Physical activity & Sleep quality & Cognitive functioning \\
\hline RI9 & Physical activity & Depressive symptoms & Social relationships \\
\hline RI10 & Physical activity & Sleep quality & Social relationships \\
\hline RI11 & Experienced stress & Depressive symptoms & Sleep quality \\
\hline RI12 & Experienced stress & Sleep quality & Depressive symptoms \\
\hline RI13 & Experienced stress & Depressive symptoms & Social relationships \\
\hline RI14 & Social relationships & Depressive symptoms & Cognitive functioning \\
\hline RI15 & Depressive symptoms & Sleep quality & Social relationships \\
\hline RI16 & Depressive symptoms & Social relationships & Sleep quality \\
\hline
\end{tabular}

Direct reinforcing loops (RD) consist of two variables and the indirect reinforcing loops (RI) consist of three variables 
(Butterfield and Halliwell 2019). This could lead to circadian misalignment when it includes damage to melanopsin-expressing retinal ganglion cells (Feng et al. 2016) and could thereby result in exacerbated oxidative stress (Musiek and Holtzman 2016).

Loops with more than three variables also exist and, although they are sometimes difficult to recognize, they may have important effects on the system (Kenzie et al. 2018). These loops can often exist between variables at multiple spatial or temporal scales and may be related to various scientific domains. An example of a longer cross-scale loop is shown in Fig. 3. A disturbance of sleep quality can lead to an impairment of glymphatic system functioning (Rasmussen et al. 2018). This may aggravate amyloid-beta as well as tau pathology (Rasmussen et al. 2018), potentially leading to neuronal dysfunction and ensuing reductions in sleep quality (Wang and Holtzman 2020).

\section{Example: Physical activity}

Physical activity is a good example of how risk factors can exert their influence on the system through the feedback loops they are involved in. Physical activity is a well-established modifiable risk factor (Livingston et al. 2017) and has high centrality in the CLD. In Fig. 4, physical activity is highlighted in the CLD with each connection, variable, and feedback loop that it is connected to. Physical activity is part of seven indirect and two direct reinforcing feedback loops, one with motor function (RD6) and one with depressive symptoms (RD7).

Increasing physical activity may reduce the onset of multiple morbidities (e.g., diabetes and obesity) by improving cardiovascular and metabolic health (Gallaway et al. 2017) and, in turn, reduce the occurrence of multimorbidity, all of which may prevent corresponding reductions in motor function (Calderón-Larrañaga et al. 2019) and thereby result in more physical activity through RI4 as well as RD6. Increasing physical activity might also reduce depressive symptoms by increasing brain-derived neurotrophic factor levels (RD7) (Mandolesi et al. 2018). These reductions in depressive symptoms could also lead to improved social relationships (VanderWeele et al. 2012), which can further stimulate physical activity (Barth et al. 2010) (RI9). Loops like RD7, which are nested in longer loops, could induce further unexpected nonlinear effects on the system.

Physical activity is also part of two cross-scale loops that involve cognitive functioning. Increasing physical activity may have beneficial effects on brain perfusion (RI5) (Mandolesi et al. 2018) as well as neuronal connectivity (RI2) via FNDC5/irisin improving synaptic plasticity (Lourenco et al. 2019), both of which can have beneficial effects on cognitive functioning (Ogoh 2017; Koen and Rugg 2019). Crossscale loops often include variables that are not only separated in space but often also in time. For example, it could take many years before a gradual loss of

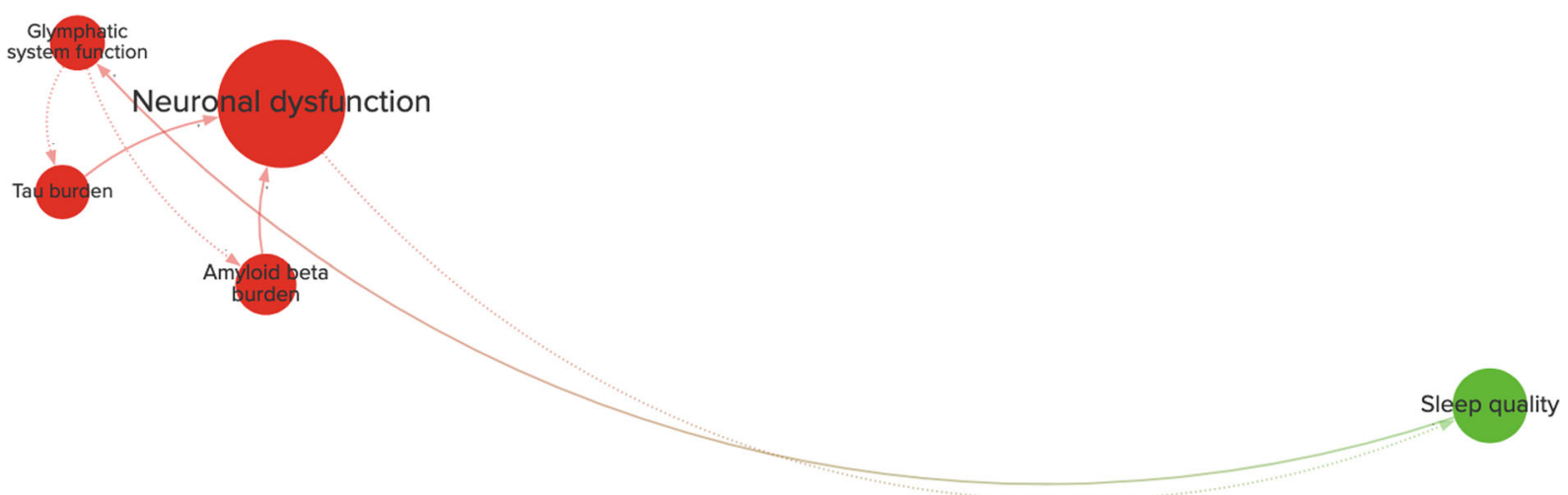

Fig. 3 An example of a reinforcing cross-scale feedback loop with four variables (from Fig. 1). The diagram is divided into variables related to brain health (red) and psychosocial health (green). A positive connection (+, solid line) represents an effect in the same direction, whereas a negative connection (-, dotted line) represents an effect in the opposite direction 


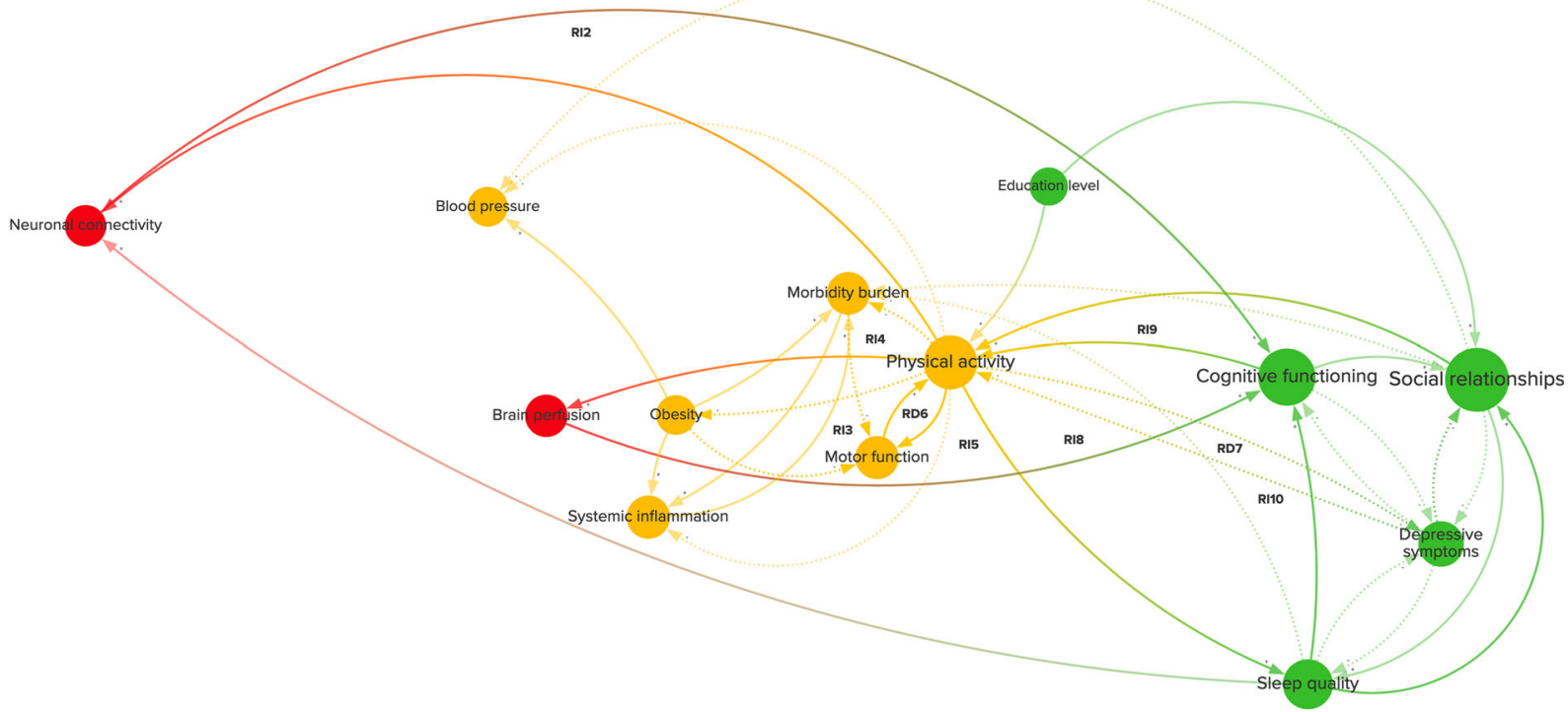

Fig. 4 Feedback loops (from Fig. 1) that include physical activity. This diagram shows the variables, causal connections, and reinforcing direct (RD6-7) and indirect (RI2-5, RI8-10) feedback loops related to physical activity. The diagram is divided into variables related to brain health (red), physical health

neuronal connectivity results in a notable loss of cognitive functioning (Koen and Rugg 2019). Loops like RI2 could, nevertheless, significantly influence AD onset and progression over such time scales.

Besides its bidirectional relationship with depressive symptoms (R7), physical activity is also closely related to other potentially modifiable factors with high centrality, specifically social relationships, and sleep quality (RI10). Figure 5 shows that depressive symptoms are involved in many feedback loops, such as with cognitive functioning (RD11), sleep quality (RD12), experienced stress (RD10), and social relationships (RD13), which also have loops amongst each other (RD9, RD14). This strong interconnection is also apparent in several indirect loops (RI15-16, RI11-12), in which these direct loops are nested, and even longer overlapping loops, such as between physical activity, sleep quality, social relationships, and depressive symptoms (Fig. 5). Such longer, crossscale loops, containing variables with high centrality and having several loops nested within them, may be crucial for understanding the onset of $\mathrm{AD}$ as well as the influence of potentially modifiable risk factors. (yellow), and psychosocial health (green). A positive connection $(+$, solid line) represents an effect in the same direction, whereas a negative connection (-, dotted line) represents an effect in the opposite direction

\section{Discussion}

CLDs can be used as a system-wide map of the multicausality of risk factors and pathophysiology of complex biomedical issues (Wittenborn et al. 2016; Kenzie et al. 2018). We have utilized this tool to summarize expert knowledge on the multicausal etiology of sporadic AD across scientific disciplines. The CLD contains variables and causal relationships formulated in a manner that this interdisciplinary group of experts agreed on. This paves the way for intensified interdisciplinary collaboration within the AD field and shows that GMB can be utilized for eliciting, contrasting, and integrating knowledge from $\mathrm{AD}$ experts across various scientific domains.

Although its qualitative nature makes it difficult to draw definite conclusions, the current CLD may already have several implications. The $\mathrm{BC}$ and $\mathrm{CC}$ point at a significant role for modifiable risk factors in AD. High centrality makes overall effects on cognitive decline via such factors more likely to occur and also underscores that such factors are endogenous to the disorder and bidirectionally related to under- 


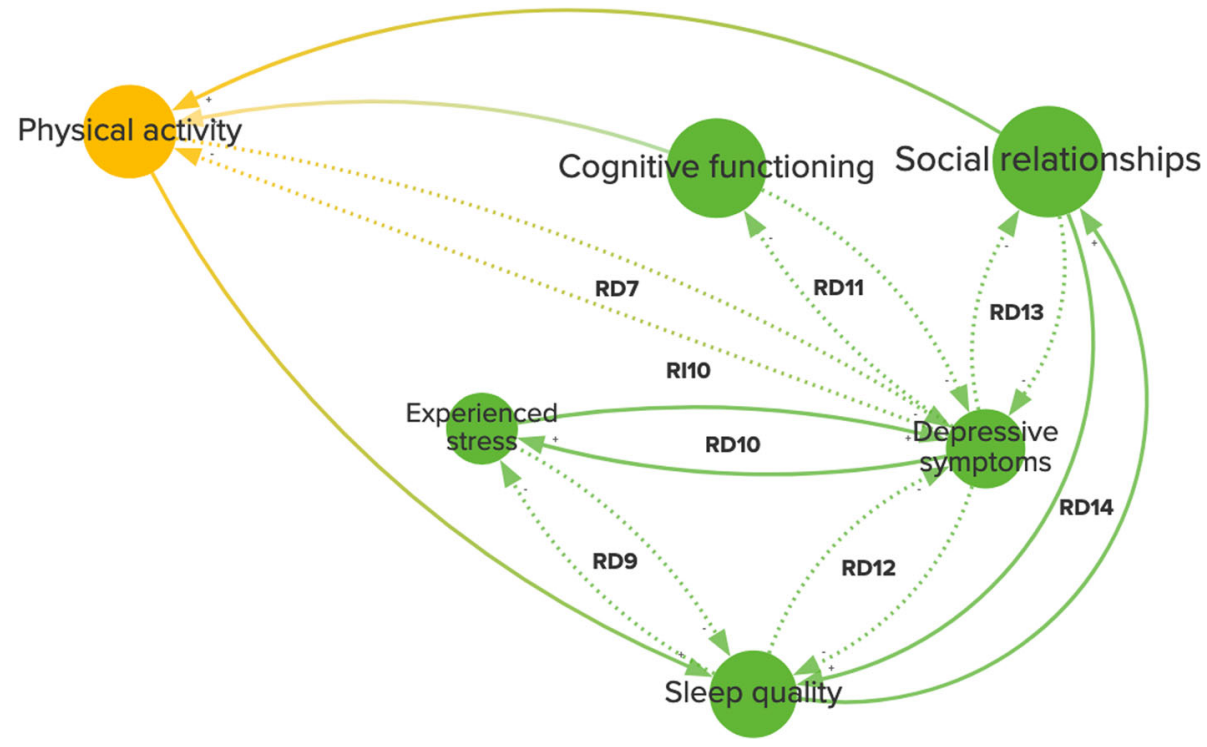

Fig. 5 A cluster of long and nested feedback loops in the causal loop diagram (Fig. 1). This diagram shows variables, causal connections, and reinforcing feedback loops related to physical health (yellow) and psychosocial health (green). A positive

lying pathophysiological processes. This supports the idea that multi-domain lifestyle interventions may be promising, particularly when intervening on social relationships, physical activity, diet, sleep, and depression. Their high centrality suggests that they mediate and impact on numerous other mechanisms relevant to AD. This is supported by results from the 2-year Finnish Geriatric Intervention Study to Prevent Cognitive Impairment and Disability (FINGER) study, which suggests that multi-domain interventions may maintain cognitive functioning in at-risk elderly people (Ngandu et al. 2015). Furthermore, the relatively high BC of cerebral endothelial dysfunction supports the increased recognition of the role of cerebrovascular dysfunction in AD and the impact of systemic vascular health on brain health (Sweeney et al. 2019).

At the very least, the CLD emphasizes the necessity of widely recognizing $\mathrm{AD}$ as a complex and multicausal condition. The many relations between the multitude of variables in the system could lead to unexpected results when intervening in them. It appears unlikely that a few variables or a single feedback loop can be isolated from the rest of the system and fully understood independently. For instance, potentially beneficial effects of an intervention may be compensated by adverse effects materializing along another pathway. It also demonstrates the need to connection (+, solid line) represents an effect in the same direction, whereas a negative connection (-, dotted line) represents an effect in the opposite direction

develop such CLDs: it is highly unlikely that an individual domain expert has an equally encompassing mental map as the resulting systemic CLD. The multicausality as illustrated by the CLD poses challenges to analysis methods that are purely based on statistical associations, such as structural equation models (Shen et al. 2020). It reveals the need for a causal structural understanding of $\mathrm{AD}$ as a system of intersecting causal pathways and loops, suggesting that there may not even be a single, localized intervention that can counter the disease progression by itself.

Importantly, the CLD approach to knowledge synthesis we utilized here is the basis for a computational implementation in the form of a system dynamics model. As a next step of the empirical cycle (Hoekstra et al. 2019), it could be used to simulate a variety of intervention scenarios on modifiable risk factors over time, which may then be tested experimentally. Rather than going immediately from theory and knowledge integration to experimental validation, simulation modeling should allow for a better understanding of the mechanisms underlying intervention scenarios and could thereby help identify potential leverage points in the system. This increased understanding may increase the prior probability of such scenarios and limit the number of failed empirical intervention studies (Ioannidis 2005, 2016). Given the 
large number of unsuccessful clinical trials (Cummings et al. 2019), this may be very important indeed.

The presence of cross-scale feedback loops further indicates a need for sustained interdisciplinary collaborations that consider long-range interactions between variables typically associated with different scientific disciplines. Many cross-scale feedback loops that can be found in Fig. 1 are longer than three variables, such as the loop in Fig. 5. Longer loops are not considered in Table 1 because they are influenced by so many variables that their interpretation becomes ambiguous without quantitative information. Such loops could nevertheless turn out to be critical to the behavior of the system in a quantitative analysis.

Despite our systematic application of GMB, there is subjectivity involved in the process. The CLD is to some extent unique to this specific group of experts and may not be easily replicated in a different group. However, the ordering of the centrality measures of the variables was robust as evidenced by the error bars in Fig. 2, which rarely overlapped. This suggests that small errors made in the wiring of the diagram would not have resulted in different qualitative conclusions, especially for the variables with high centrality. Furthermore, due to the wide range of expertise included in our group and the embedding of all connections in the scientific literature, such errors are likely minimized. To assess the influence of bias in the variable selection on the centrality measures, we used cerebrovascular pathology as an example and tested whether omitting white matter hyperintensities, microbleeds, and lacunar infarcts from the CLD would alter the BC ranking of cerebral endothelial dysfunction. This test yielded minimal changes and only slightly lowered the $\mathrm{BC}$ of endothelial dysfunction to a comparable level as cognitive functioning. Limiting the level of detail was a deliberate choice by the group and resulted in a more comprehensible CLD. We aimed to include the most important mechanisms that contribute to $\mathrm{AD}$ rather than to be completely exhaustive. Consequently, several processes have been combined into aggregate variables. For example, cellular processes like mitochondrial dysfunction and endoplasmic reticulum stress have been aggregated in overarching terms such as neuronal dysfunction. Questions at higher levels of detail can thus not yet be addressed using the current CLD. Future efforts could extend the CLD by increasing its level of detail, particularly at the (sub-)cellular scale.
A pitfall of CLDs is that they exclusively contain qualitative information. Accordingly, the centrality measures of the variables might change considerably when taking into account the strength of the connections. The use of centrality measures for causal importance has also been disputed recently (van Elteren and Quax 2019; Dablander and Hinne 2019) since it does not take into account the specific dynamics represented by the variables and connections. That being said, high centrality variables were found to correspond to known drivers of childhood obesity in a CLD (McGlashan et al. 2016). Therefore, as a first approximation and while dynamics are not yet modeled quantitatively, variables with high centrality might—on the whole-be responsible for a significant proportion of the effect on cognitive decline in our CLD as well. At this point, a network-structural approach for proposing causally relevant variables is the best we can do. The moderate-to-large monotonic correlation between BC and $\mathrm{CC}$ further suggests that, although they overlap, these measures also have complementary value. For example, $\mathrm{CC}$ was useful for comparing the exogenous variables.

The CLD remains a reflection of the knowledge of our group of experts and of the available scientific evidence. Hence, some connections that exist in reality may have been omitted in the CLD. The implications of this uncertainty will be assessed using uncertainty quantification (UQ) techniques applied to the system dynamics model. Because our CLD is the result of GMB and our review of scientific literature was not fully systematic, the CLD by itself does not yet provide insight into which of the connections require further investigation. However, when computationally implemented, the CLD could become a valuable, complementary tool to systematic reviews for identifying potentially interesting empirical research targets. In the sequel to this work, UQ and sensitivity analysis techniques applied to the system dynamics model will be used to identify which of the connections are important for model predictions and which would require additional data in order to be estimated with high confidence. Through these means, we will investigate which parts of the model require further scientific research. The lack of balancing feedback loops is likely a consequence of our aim of mapping the mechanisms leading to cognitive decline in $\mathrm{AD}$, rather than homeostatic and resilience mechanisms. For a fuller picture, future efforts could also aim to chart out 
balancing loops to give a more complete representation of the system that is less skewed to factors that promote the development and progression of clinically manifest $\mathrm{AD}$ and instead also includes factors that counteract mechanisms of disease progression.

Although the CLD is, just like any other model (Sterman 2002), an incomplete representation of reality, it can still be used for educational purposes, hypothesis generation, and the identification of confounder and collider variables to consider in statistical analyses (VanderWeele 2019). Furthermore, the CLD is the first step in an iterative process of not only developing computational models but also further mapping out the processes implicated in AD. As such, it could become increasingly exhaustive, reaching levels of detail like the Foresight system map developed for obesity (Vandenbroeck et al. 2007).

We have the ambition of turning this work into an ongoing effort of the scientific community to expand and parameterize the model we developed, but a rigorous methodology for such a communitylevel approach must still be developed. For now, we encourage $\mathrm{AD}$ researchers to use our online version of the CLD to make suggestions for improvements or extensions using the designated comment function. Additional systematic methods for triangulation and extending the CLD might entail word cloud analyses (Atenstaedt 2012) in order to identify important variables that are not currently present in the diagram, or systemic reviews which could be conducted for every connection that might plausibly exist between any of the variables in the CLD. In addition, quality assessment criteria could be developed for the application of GMB to complex scientific issues, similar to the PRISMA criteria for systematic reviews (Moher et al. 2009). This would promote the quality and wide-scale reliable application of GMB and of mixed quantitative and qualitative methods.

\section{Conclusion}

This work demonstrates the relevance and feasibility of using systems thinking and GMB to improve our understanding of the biopsychosocial causality in AD. Our study specifically serves as a proof-of-concept of this innovative method in the AD field. The general notion that $\mathrm{AD}$ is complex and multicausal is supported by the large number of variables, causal connections, and feedback loops that we have identified in the system, which exist at multiple spatial and time scales. As such, this work is complementary to scientific approaches confined to a single mechanism that cannot fully account for the interactive behavior of the network of multiple causes at different scales.

We will use the CLD for the development of a system dynamics model. This model will be calibrated and validated using empirical data of longitudinal cohort studies and randomized clinical trials. By these means, it may become an important tool for simulating and predicting cognitive and global functional performance effects of multi-domain lifestyle or drug interventions. We believe that GMB and systems thinking should be further developed for and applied to complex multicausal conditions, particularly AD.

Funding information This project was partly granted as GEENA-Q-19-595225 by the American Alzheimer's Association to M. Olde Rikkert and an unrestricted grant of the research funds from Clinical Pharmacologist Prof. Dr. JM v Rossum, Nijmegen, The Netherlands.

Additional funding sources: Eddy van der Zee and Marcel Olde Rikkert: Deltaplan Dementie; Memorabel Study, funded by The Netherlands Organisation for Health Research and Development (project number 733050303).

Eddy van der Zee, Isabelle van der Velpen and René Melis: Deltaplan Dementie; Memorabel Study, funded by The Netherlands Organisation for Health Research and Development (project number 733050831).

Jeroen de Bresser: Alzheimer Nederland Grant (WE.032019-08)

Hieab H. H. Adams: ZonMW (grant number 916.19.151).

Inge M.C.M. de Kok: Funding from the Dutch Research Council (NWO, Grant 016.Veni.198.020).

Rick Quax acknowledges ZonMw project DINAMICS (project number 531003015), as well as Horizon 2020 project ToAition (grant number 848146).

Marcel Verbeek: Deltaplan Dementie; BIONIC project, Memorabel Study, funded by The Netherlands Organisation for Health Research and Development (project number 733050822).

Data availability A supplementary excel file is available for this work and an online version of the CLD is provided https://cldforad.kumu.io/mapping-the-complex-multicausalityin-alzheimers-disease?token=16CvrnWTeDcLkHR1.

\section{Compliance with ethical standards}

Conflict of interest The authors declare that they have no conflict of interest.

Ethics approval Not applicable. 
Consent to participate All participating experts are coauthors of this work .

Open Access This article is licensed under a Creative Commons Attribution 4.0 International License, which permits use, sharing, adaptation, distribution and reproduction in any medium or format, as long as you give appropriate credit to the original author(s) and the source, provide a link to the Creative Commons licence, and indicate if changes were made. The images or other third party material in this article are included in the article's Creative Commons licence, unless indicated otherwise in a credit line to the material. If material is not included in the article's Creative Commons licence and your intended use is not permitted by statutory regulation or exceeds the permitted use, you will need to obtain permission directly from the copyright holder. To view a copy of this licence, visit http:// creativecommonshorg/licenses/by/4.0/.

\section{References}

Ahmed SE (2017) Big and complex data analysis: methodologies and applications. Springer, Berlin

Alhola P, Polo-Kantola P (2007) Sleep deprivation: impact on cognitive performance. Neuropsychiatric disease and treatment 3(5):553-567

Andersen DF, Vennix JA, Richardson GP, Rouwette EA (2007) Group model building: problem structuring, policy simulation and decision support. J Oper Res Soc 58(5):691-694

Atenstaedt R (2012) Word cloud analysis of the BJGP. Br J Gen Pract 62(596):148-148

Bala BK, Arshad FM, Noh KM (2017) Causal loop diagrams. Springer, Berlin, pp 37-51

Barth J, Schneider S, Von Känel R (2010) Lack of social support in the etiology and the prognosis of coronary heart disease: a systematic review and meta-analysis. Psych Med 72(3):229-238

Braithwaite J, Churruca K, Long JC, Ellis LA, Herkes J (2018) When complexity science meets implementation science: a theoretical and empirical analysis of systems change. BMC Med 16(1):63

Brandes U, Borgatti SP, Freeman LC (2016) Maintaining the duality of closeness and betweenness centrality. Soc Netw 44:153-159

Bérard C (2010) Group model building using system dynamics: an analysis of methodological frameworks. J Bus Res 8(1):13-24

Butterfield DA, Halliwell B (2019) Oxidative stress, dysfunctional glucose metabolism and Alzheimer disease. Nat Rev Neurosci 20(3):148-160

Calderón-Larrañaga A, Vetrano DL, Ferrucci L, Mercer SW, Marengoni A, Onder G, Eriksdotter M, Fratiglioni L (2019) Multimorbidity and functional impairment-bidirectional interplay, synergistic effects and common pathways. J Internal Med 285(3):255-271

Corley J, Cox SR, Harris SE, Hernandez MV, Maniega SM, Bastin ME, Wardlaw JM, Starr JM, Marioni RE, Deary IJ (2019) Epigenetic signatures of smoking associate with cognitive function, brain structure, and mental and physical health outcomes in the lothian birth cohort 1936. Transl Psych 9(1):1-15

Cummings J, Lee G, Ritter A, Sabbagh M, Zhong K (2019) Alzheimer's disease drug development pipeline: 2019. Alzheimer's \& Dementia: Transl Res Clin Intervent 5:272293

Dablander F, Hinne M (2019) Node centrality measures are a poor substitute for causal inference. Sci Rep 9(1):1-13

Dorszewska J, Prendecki M, Oczkowska A, Dezor M, Kozubski W (2016) Molecular basis of familial and sporadic Alzheimer's disease. Curr Alzheimer Res 13(9):952963

Feng R, Li L, Yu H, Liu M, Zhao W (2016) Melanopsin retinal ganglion cell loss and circadian dysfunction in Alzheimer's disease. Mol Med Rep 13(4):3397-3400

Forrester JW (1971) Counterintuitive behavior of social systems. Theory Decis 2(2):109-140

Forrester JW (2009) Some basic concepts in system dynamics. Sloan School of Management. Massachusetts Institute of Technology, Cambridge, p 9

Fotuhi M, Hachinski V, Whitehouse P (2009) Paradigm shift from Alzheimer-centric view of brain aging and dementia: dynamic polygon hypothesis. Alzheimer's Dement 5(4):275-276

Gallaway PJ, Miyake H, Buchowski MS, Shimada M, Yoshitake Y, Kim AS, Hongu N (2017) Physical activity: a viable way to reduce the risks of mild cognitive impairment, Alzheimer's disease, and vascular dementia in older adults. Brain Sci 7(2):22

Gustafson DH, Delbecq AL, Van de Ven AH (1986) Group techniques for program planning-a guide to nominal group and Delphi processes. Group Organ Stud 1(2):256-256

Hardy J, Selkoe DJ (2002) The amyloid hypothesis of Alzheimer's disease: progress and problems on the road to therapeutics. Science 297(5580):353-356

Heneka MT, Carson MJ, El Khoury J, Landreth GE, Brosseron F, Feinstein DL, Jacobs AH, Wyss-Coray T, Vitorica J, Ransohoff RM et al (2015) Neuroinflammation in Alzheimer's disease. Lancet Neurol 14(4):388-405

Hoekstra AG, Chopard B, Coster D, Portegies Zwart S, Coveney PV (2019) Multiscale computing for science and engineering in the era of exascale performance. Phil Trans R Soc A 377:20180144

Hovmand PS, Andersen DF, Rouwette E, Richardson GP, Rux K, Calhoun A (2012) Group model-building 'scripts' as a collaborative planning tool. Syst Res Behav Sci 29(2):179_ 193

Ioannidis JP (2005) Why most published research findings are false. PLoS Med 2(8):e124

Ioannidis JP (2016) Why most clinical research is not useful. PLoS Med 13(6):e1002049

Karran E, Mercken M, De Strooper B (2011) The amyloid cascade hypothesis for Alzheimer's disease: an appraisal for the development of therapeutics. Nat Rev Drug Discov 10(9):698-712

Kenzie ES, Parks EL, Bigler ED, Wright DW, Lim MM, Chesnutt JC, Hawryluk GWJ, Gordon W, Wakeland W (2018) The dynamics of concussion: mapping pathophysiology, persistence, and recovery with causal-loop diagramming. Front Neurol 9(4):203 
Koen JD, Rugg MD (2019) Neural dedifferentiation in the aging brain. Trends Cogn Sci 23(7):547-559

Kuljis RO (2009) Toward a multi-dimensional formulation of the pathogenesis and pathophysiology of the Alzheimer dementia-like syndrome applicable to a variety of degenerative disorders and normal cognition. Med Hyp 73(3):315318

Kumu (2019) https://kumu.io/, accessed 11 October 2019

Littlejohns LB, Baum F, Lawless A, Freeman T (2018) The value of a causal loop diagram in exploring the complex interplay of factors that influence health promotion in a multisectoral health system in Australia. Health Res Policy Syst 16(1):126

Livingston G, Sommerlad A, Orgeta V, Costafreda SG, Huntley J, Ames D, Ballard C, Banerjee S, Burns A, CohenMansfield J et al (2017) Dementia prevention, intervention, and care. Lancet 390(10113):2673-2734

Lourenco MV, Frozza RL, de Freitas GB, Zhang H, Kincheski GC, Ribeiro FC, Gonċalves RA, Clarke JR, Beckman D, Staniszewski A et al (2019) Exercise-linked FNDC5/irisin rescues synaptic plasticity and memory defects in Alzheimer's models. Nat Med 25(1):165

Mandolesi L, Polverino A, Montuori S, Foti F, Ferraioli G, Sorrentino P, Sorrentino G (2018) Effects of physical exercise on cognitive functioning and wellbeing: biological and psychological benefits. Front Psychol 9:509

Marais AD (2019) Apolipoprotein E in lipoprotein metabolism, health and cardiovascular disease. Pathology 51(2):165176

McGlashan J, Johnstone M, Creighton D, de la Haye K, Allender S (2016) Quantifying a systems map: network analysis of a childhood obesity causal loop diagram. PloS One 11(10):e0165459

McKhann GM, Knopman DS, Chertkow H, Hyman BT, Jack JrCR, Kawas CH, Klunk WE, Koroshetz WJ, Manly JJ, Mayeux R, Mohs RC, Morris JC, Rossor MN, Scheltens P, Carrillo MC, Thies B, Weintraub S, Phelps CH (2011) The diagnosis of dementia due to Alzheimer's disease: recommendations from the National Institute on Aging-Alzheimer's Association workgroups on diagnostic guidelines for Alzheimer's disease. Alzheimers Dement 7(3):263-269

Moher D, Liberati A, Tetzlaff J, Altman DG (2009) Preferred reporting items for systematic reviews and meta-analyses: the PRISMA statement. Ann Internal Med 151(4):264269

Mortimer JA (2012) The nun study: risk factors for pathology and clinical-pathologic correlations. Curr Alzheimer Res 9(6):621-627

Musiek ES, Holtzman DM (2016) Mechanisms linking circadian clocks, sleep, and neurodegeneration. Science 354(6315):1004-1008

Newcombe EA, Camats-Perna J, Silva ML, Valmas N, Huat TJ, Medeiros R (2018) Inflammation: the link between comorbidities, genetics, and Alzheimer's disease. J Neuroinflammation 15(1):1-26

Ngandu T, Lehtisalo J, Solomon A, Levälahti E, Ahtiluoto S, Antikainen R, Bäckman L, Hänninen T, Jula A, Laatikainen $\mathrm{T}$ et al (2015) A 2 year multidomain intervention of diet, exercise, cognitive training, and vascular risk monitoring versus control to prevent cognitive decline in at-risk elderly people (FINGER): a randomised controlled trial. Lancet 385(9984):2255-2263

Nunomura A, Castellani RJ, Zhu X, Moreira PI, Perry G, Smith MA (2006) Involvement of oxidative stress in Alzheimer disease. J Neuropathol Exper Neurol 65(7):631-641

Ogoh S (2017) Relationship between cognitive function and regulation of cerebral blood flow. J Physiol Sci 67(3):345-351

Pimplikar SW (2009) Reassessing the amyloid cascade hypothesis of Alzheimer's disease. Int J Biochem Cell Biol 41(6):1261-1268

Pomorska G, Ockene JK (2017) A general neurologist's perspective on the urgent need to apply resilience thinking to the prevention and treatment of Alzheimer's disease. Alzheimer's \& Dementia: Transl Res Clin Intervent 3(4):498-506

Rasmussen MK, Mestre H, Nedergaard M (2018) The glymphatic pathway in neurological disorders. Lancet Neurol 17(11):1016-1024

Rock P, Roiser J, Riedel W, Blackwell A (2014) Cognitive impairment in depression: a systematic review and metaanalysis. Psychol Med 44(10):2029-2040

Rollo JL, Banihashemi N, Vafaee F, Crawford JW, Kuncic Z, Holsinger RD (2016) Unraveling the mechanistic complexity of Alzheimer's disease through systems biology. Alzheimer's Dement 12(6):708-718

Savva GM, Wharton SB, Ince PG, Forster G, Matthews FE, Brayne C (2009) Medical Research Council Cognitive Function and Ageing Study Age, neuropathology, and dementia. N Engl J Med 360(22):2302-2309

Shen X, Ma S, Vemuri P, Simon G (2020) Challenges and opportunities with causal discovery algorithms: application to Alzheimer's pathophysiology. Sci Rep 10(1):1-12

Sterman J (2000) Business dynamics: systems thinking and modeling for a complex world. Irwin/McGraw-Hill, New York

Sterman JD (2002) All models are wrong: reflections on becoming a systems scientist. Syst Dyn Rev 18(4):501-531. https://doi.org/10.1002/sdr.261

Sweeney MD, Montagne A, Sagare AP, Nation DA, Schneider LS, Chui HC, Harrington MG, Pa J, Law M, Wang DJ et al (2019) Vascular dysfunction-the disregarded partner of Alzheimer's disease. Alzheimer's Dement 15(1):158-167

Tang Y, Lutz MW, Xing Y (2019) A systems-based model of Alzheimer's disease. Alzheimer's Dement 15(1):168-171

Tejera D, Heneka MT (2016) Microglia in Alzheimer's disease: the good, the bad and the ugly. Curr Alzheimer Res 13(4):370-380

van Dijk G, van Heijningen S, Reijne AC, Nyakas C, van der Zee EA, Eisel UL (2015) Integrative neurobiology of metabolic diseases, neuroinflammation, and neurodegeneration. Front Neurosci 9:173

van Elteren C, Quax R (2019) The dynamic importance of nodes is poorly predicted by static topological features. arXiv: 190406654

Vandenbroeck P, Goossens J, Clemens M (2007) Foresight: tackling obesities: future choices - building the obesity system map. PsycEXTRA Dataset

VanderWeele TJ, Hawkley LC, Cacioppo JT (2012) On the reciprocal association between loneliness and subjective well-being. Am J Epidemiol 176(9):777-784

VanderWeele TJ (2019) Principles of confounder selection. Eur J Epidemiol 34(3):211-219 
Vennix JA (1996) Group model building: facilitating team learning using system dynamics field. Wiley, New York

Vennix JA (1999) Group model-building: tackling messy problems. Syst Dyn Rev J Syst Dyn Soc 15(4):379401

Vensim (2019) https://vensim.com, accessed 30 August 2019

Wallace LMK, Theou O, Godin J, Andrew MK, Bennett DA, Rockwood K (2019) Investigation of frailty as a moderator of the relationship between neuropathology and dementia in Alzheimer's disease: a cross-sectional analysis of data from the rush memory and aging project. Lancet Neurol 18(2):177-184

Wang C, Holtzman DM (2020) Bidirectional relationship between sleep and Alzheimer's disease: role of amyloid, tau, and other factors. Neuropsychopharmacology 45(1):104120
Wilson R, Hebert L, Scherr P, Barnes L, De Leon CM, Evans D (2009) Educational attainment and cognitive decline in old age. Neurology 72(5):460-465

Wittenborn AK, Rahmandad H, Rick J, Hosseinichimeh N (2016) Depression as a systemic syndrome: mapping the feedback loops of major depressive disorder. Psychol Med 46(3):551-562

Xu K, Montalvo-Ortiz JL, Zhang X, Southwick SM, Krystal JH, Pietrzak RH, Gelernter J (2019) Epigenome-wide dna methylation association analysis identified novel loci in peripheral cells for alcohol consumption among European American male veterans. Alcoholism: Clin Exper Res 43(10):2111-2121

Publisher's note Springer Nature remains neutral with regard to jurisdictional claims in published maps and institutional affiliations. 